\title{
Reaktionen, Regulationen und Adaptationen der Tiere nach Veränderung von Temperatur und Salzgehalt. Versuche mit Zoothamnium biketes (Ciliata, Peritricha)
}

\author{
Herbert Precht und Elke Lindner \\ Zoologisches Institut der Universität Kiel, Kiel \\ Lebrstwbl für vergleichende Physiologie und Tierpsychologie
}

\begin{abstract}
Responses, regulations and adaptations of animals following changes in temperature and salinity. Experiments with Zootbamnium biketes(Ciliata, Peritricha). Upon sudden changes in external salinity the ciliate protozoon Zoothamnium hiketes PRECHT temporarily changes its cell volume. Resistance to very low and high salinities depends on the previous salinity history. The time course of changes in resistance to external salinity is described. "Aftereffects" in the sense of temperature hardening were not observed. Considering these findings, responses, regulations and adaptations (acclimatizations) of animals exposed to dhanges in temperature and salinity are discussed in general.
\end{abstract}

\section{EINLEITUNG}

Viele Tiere sind in der Natur wechselnden Temperaturen und Salzgehalten ihres Mediums ausgesetzt. Im letzten Fall verhalten sich bekanntlich poikilo- und homoiosmotische Tiere unterschiedlich; Abbildung 1 stellt einige markante Typen dar, die natürlich durch Übergänge verbunden sein können. Temperaturänderungen wirken sich auf poikilo- und homoiotherme Tiere verschieden aus. Die letzteren halten ihre Körpertemperatur konstant, was den ersteren im allgemeinen nicht möglich ist. Diese können dagegen vielfach trotz unterschiedlicher Körpertemperaturen ihren Stoffwechsel mehr oder weniger ideal auf eine Norm einstellen, was als Leistungsadaptation (capacity adaptation) bezeichnet wird. Daneben gibt es eine Resistenzadaptation gegenüber extremen Temperaturen.

Es sollen zunächst einige Versuche an Zoothamnium biketes PRECHT geschildert werden, die von E. LiNDNER ausgeführt wurden. Z. biketes ist nach Precht (1935) ein epizoisch auf Gammariden lebendes peritriches Ciliat, das bereits von MüLLER (1936) in seinem Verhalten bei verändertem Salzgehalt und von VogeL (1966) hinsichtlich seiner Fähigkeit zur thermischen Resistenzadaptation untersucht wurde (über das Vorkommen des Ciliaten vgl. auch FENCHEL 1965). Die Ergebnisse der Versuche an diesem Einzeller sollen mitbenutzt werden, um ganz allgemein die Reaktionen, Regulationen und Adaptationen (= Akklimatisationen) nach Veränderung der Temperatur und des Salzgehaltes des Außenmediums zu vergleichen. 


\section{MATERIAL UND METHODIK}

Unsere Versuchstiere entstammten der Kieler Förde. Z. hiketes gehört wahrscheinlich zum Typ 2 der Abbildung 1, da das Epizoon sowohl in der Kieler Förde (Salzgehalt 13 bis $18 \%$, maximal 26,6\%, minimal 8,8\%; OHL 1959) und an den Fangstellen von Fenchel (1965) (Ostsee, Oresund, Salzgehalt bis $35 \%$ ) als auch in der

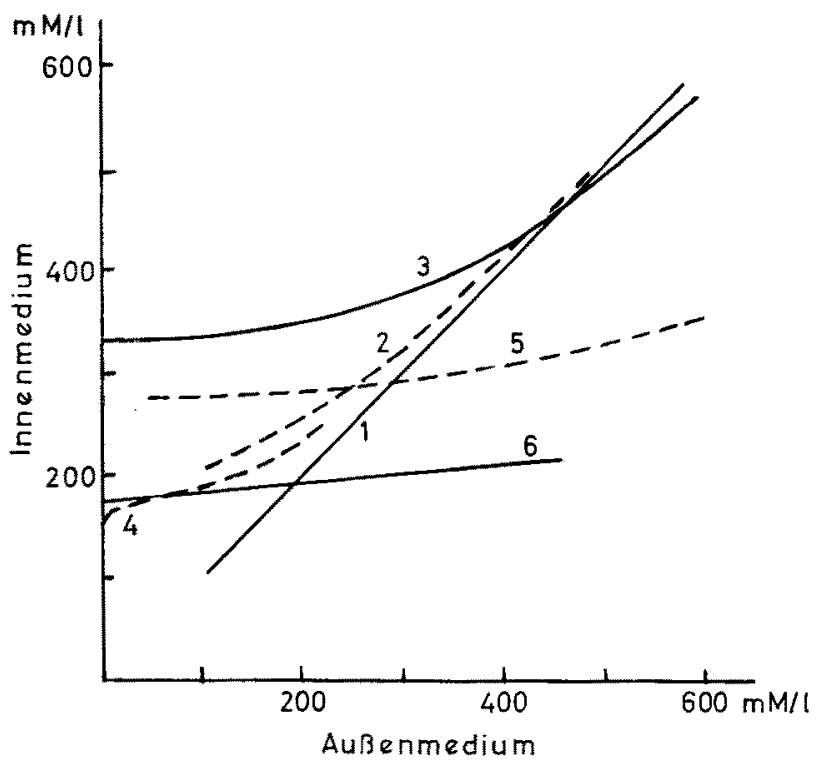

Abb. 1: Beziehungen zwischen den osmotischen Konzentrationen des Innen- und Außenmediums bei einigen Tieren, die verschiedene Typen repräsentieren. 1: Mytilus edulis, 2: Nereis diversicolor, 3: Eriocheier sinensis, 4: Gammarus pulex, 5: Palaemonetes varians (dringt in einigen Rassen bis ins Süßwasser vor), 6: Anguilla wulgaris. (Nach mehreren Autoren; vgl. Remane \& SCHLIEPER 1958)

Schlei bei Schleswig (2,5 bis $8 \%$; NelLeN 1963) vorkommt. Bei den Versuchen von Vogel (1966) wurde ein Salzgehalt von 3 bis $30 \%$ toleriert. Nach der Definition von Ax \& Ax (1960) handelt es sich um ein marin-euryhalines Tier.

Die Krebse mit ihren Epizoen zwischen den Borsten der Urosomsegmente und an den Gelenken der hinteren Pereiopoden wurden für mindestens 7 (meistens 11) Tage in Plastikeimern (10 1 Inhalt) an folgende Salzgehalte (AS) angepaßt: 5,3\% beziehungsweise $5,6 \% 0,19,4 \%$ und $30 \%$ (Aufbewahrung bei guter Belütung und $16 s t u ̈ n d i g e r$ Belichtung; Temperatur $6^{\circ} \mathrm{C}$ ). Den Salzgehalt bestimmten wir refraktometrisch; höhere Konzentrationen erhielten wir durch Aufsalzen von Fördewasser mit Meersalz, niedrigere durch Verdünnen mit Leitungswasser. Während der Versuche konnten die Zoothamnien in einem kleinen Versuchsbecken beobachtet werden, das von der Flüssigkeit eines Colora-Kühlaggregats umspült wurde (Versuchstemperatur $6^{\circ} \mathrm{C}$ ). 


\section{UNTERSUCHUNGSERGEBNISSE}

Setzten wir die Zoothamnien von einem Salzgehalt von $30 \%$ in $5 \%$ um, so nahm deren Zellvolumen deutlich $\mathrm{zu}$, im umgekehrten Fall verringerte es sich. Erst nach einigen Stunden war die normale Größe wieder erreicht (wir haben die Volumenänderungen nicht zahlenmäßig erfaßt). Andere Protozoen verhalten sich ähnlich, etwa Paramecium (Frisch 1944), Amoeba (Hopkns 1946) und Zoothamnien. Prosser weist nach einer Literaturbesprechung darauf hin, daß bei Protozoen of sogar Wasser langsam permeiert (Prosser \& Brown 1961, p. 21). Für einen sehr langsamen Ausgleich der Konzentration nach Salzgehaltsänderungen sprechen die Messungen von OberTHÜr (1937) an Frontonia marina aus einer Binnenland-Salzquelle. Tiere, die einige Tage in Wasser mit verschiedenem Salzgehalt gelebt hatten, zeigten etwa die gleiche Pumpfrequenz der kontraktilen Vakuole; dies gilt überraschenderweise auch für salzloses Wasser, in dem die Zellen hypertonisch sein müssen; doch mag das damit zusammenhängen, daß dieser Bereich für $F$. marina unbiologisch ist. Bei raschen Anderungen des Salzgehaltes tritt sehr wohl eine $\mathrm{Zu}$ - beziehungsweise Abnahme der Pumpfrequenz der Vakuole ein. Die konstante Pumpfrequenz nach erfolgter Angleichung hat nach OBERTHüR im wesentlichen andere als osmoregulatorische Aufgaben zu erfüllen. Wasser tritt bei diesem Tier somit relativ schnell ein oder aus, was dann die Anderung der Schlagfrequenz bedingt, bis der Konzentrationsausgleich beendet ist.

Bei den Versuchen von Müller (1936) mit Z. biketes nahm die von der Vakuole nach außen gepumpte Wassermenge mit abnehmendem Salzgehalt des Außenmediums zu. (Ausnahme: sehr niedrige Salzgehalte). Hierbei handelte es sich um kurzfristige Anderungen der Salzkonzentration. Auch bei unserem Versuchstier müßte untersucht werden, ob die Pumpfrequenz der Vakuole im poikilosmotischen Bereich nach einer längeren Angleichung an die verschiedenen Salzgehaltsstufen konstant wird.

Weitere Versuche galten dem Resisten z problem. Man muß dabei nicht nur den Salzgehalt während der Messungen zur Ermittlung der osmotischen Resistenz (RS), sondern auch den des Aufbewahrungswassers vor den Versuchen (AS) berücksichtigen.

Die Ermittlung der osmotischen Resistenz kann auf zwei Wegen erfolgen: Die Versuchstiere werden entweder direkt in eine Lösung definierter Konzentration überführt, oder der Salzgehalt wird mit einer konstanten Rate verändert, wobei meist höhere Resistenzwerte gemessen werden als bei der ersten Methode, die ron uns angewandt wurde. Es bleibt noch die letale Salzkonzentration zu definieren. Man kann die durchschnittliche Überlebenszeit bei einer konstanten Versuchskonzentration bestimmen oder aber den Salzgehalt (RS), bei dem ein bestimmter Prozentsatz der Tiere (meist 50\%) eine gegebene Zeit überlebt. Die Anwendung der ersten Methode stieß deshalb auf Schwierigkeiten, weil der Eintritt des Todes bei Z. biketes nicht genau feststellbar war. Setzte man die Tiere von einer mittleren in eine hohe Salzkonzentration, so kontrahierten sich die Kolonien zuerst sehr stark, zuckten dann mehrere Male mit abnehmender Intensität, bis sie ruhig aber kontrahiert blieben. Setzte man solche Tiere kurz nach der letzten Zuckung in das Ausgangsmedium zurück, so erholten sie sich. Bei einer späteren Umsetzung geschah dies jedoch nicht mehr; der nunmehr ein- 
getretene Tod war äußerlich nicht zu erkennen. Wir haben uns daher darauf beschränkt, den Prozentsatz der Tiere zu bestimmen, die einen Aufenthalt von einer halben Stunde in der jeweiligen Konzentration (RS) ïberlebten. Aus den Kurven kann der 50\%-Wert abgelesen werden.

Im einzelnen gingen wir wie folgt vor: In dem Anpassungsmedium (AS) trennten wir mit einer Schere die besiedelten Urosomsegmente oder Pereiopoden der Krebse ab und steckten sie auf eine Insektennadel. Die Zahl der Epizoen und ihr Sitz auf dem Substrat wurden notiert. Wie überführten die Tiere dann in die gewünschten Salzkonzentrationen (RS) und nach 30 Minuten wieder abrupt in das Ausgangsmedium zurück; die Registrierung der Überlebenden erfolgte frühestens eine halbe Stunde später.

Bei der Bestimmung der oberen Grenze gingen wir von einem Salzgehalt (RS) aus, bei dem alle untersuchten Tiere überlebten, und erhöhten ihn dann solange, bis alle Zoothamnien starben. Dabei zeigte sich, daß der RS, bei dem gerade $50 \%$ der Tiere überlebten, für die Zoothamnien aus $A S=19,4 \%$ einerseits niedriger ist als bei denen aus AS $=30 \%$, andererseits aber höher als bei den Tieren aus $\mathrm{AS}=$ $5,6 \%$ (Tab. 1). Die thermische Resistenzadaptation haben wir als sinnvoll (reason-

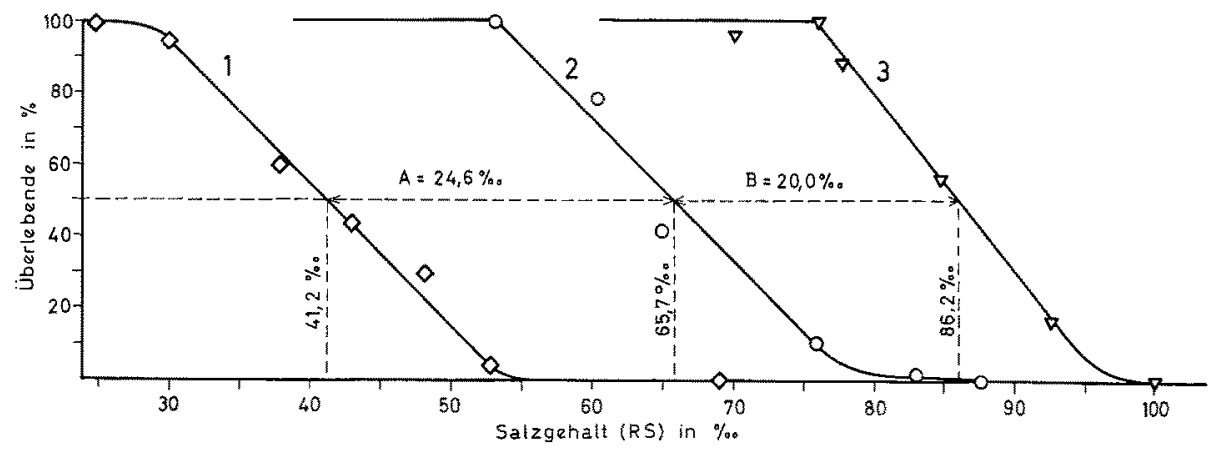

Abb. 2: Abhängigkeit der oberen osmotischen Resistenzgrenze von Z. hiketes vom Salzgehalt des Anpassungsmediums (AS). 1: AS $=5,6 \%, 2: \mathrm{AS}=19,4 \%, 3: \mathrm{AS}=30 \%$. Gestrichelte Linie: $50 \%$ Überlebende mir den Abständen $A$ und $B$ zwischen den Kurven

able) bezeichnet, wenn die warmadaptierten Tiere hitzeresistenter und kälteempfindlicher sind als die kaltadaptierten; im umgekehrten Fall liegen paradoxe Phänomene vor. Z. biketes zeigt nach dieser Nomenklatur eine sinnvolle „Anpassung“, was besonders aus Abbildung 2 ersichtlich ist. Auch die 50\%-Linie ist eingezeichnet mit den $\mathrm{Ab}$ ständen $(A, B) z$ wischen den Kurven. Die Verschiebung der oberen Resistenzgrenze ist etwa proportional zur Veränderung des Anpassungsmediums (AS).

Die unteren Grenzen liegen viel dichter zusammen, doch geht aus Tabelle 2 hervor, daß auch diese "Anpassung" sinnvoll ist. Die Unterschiede zwischen $\mathrm{AS}=5,6 \%$ und $19,4 \%$ beziehungsweise AS $=19,4 \%$ und $30 \%$ bei RS $=0 \%$ wurden mit dem $\chi^{2}$-Test abgesichert; es ergibt sich jeweils eine Irrtumswahrscheinlichkeit von $\alpha<0,1 \%$, was Signifikanz bedeutet (vgl. WeBER 1961). 
Tabelle 1

Abhängigkeit der oberen osmotischen Resistenzgrenze vom Salzgehalt des Anpassungsmediums (AS). RS = Salzgehalt während der Messungen. Eingeklammert: Anzahl der registrierten Individuen

\begin{tabular}{|c|c|c|c|}
\hline \multirow{2}{*}{ RS in $\%$} & \multicolumn{3}{|c|}{ Uberlebende in Prozent } \\
\hline & $\mathrm{AS}=5,6 \%$ & $\mathrm{AS}=19,4 \%$ & $\mathrm{AS}=30 \%$ \\
\hline 24,7 & $98,5 \quad(134)$ & & \\
\hline 30,0 & $94,1(154)$ & & \\
\hline 37,7 & $59,6(136)$ & & \\
\hline 42,8 & $43,2(183)$ & & \\
\hline 48,1 & $29,3(316)$ & & \\
\hline 52,8 & $4,1 \quad(144)$ & $99,4 \quad(157)$ & \\
\hline 60,2 & & $78,1(288)$ & \\
\hline 64,8 & & $41,5(197)$ & \\
\hline 68,8 & $0 \quad(240)$ & & \\
\hline 70,0 & & & $95,5(109)$ \\
\hline 75,8 & & $10,5(116)$ & $99,2 \quad(94)$ \\
\hline 77,6 & & & $88,0(109)$ \\
\hline 83,0 & & $1,9(105)$ & \\
\hline 84,7 & & & $56,0(102)$ \\
\hline 87,6 & & $0 \quad(152)$ & \\
\hline 92,5 & & & $16,5(108)$ \\
\hline 100,0 & & & o (115) \\
\hline 106,4 & & & $0 \quad(110)$ \\
\hline
\end{tabular}

Tabelle 2

Abhängigkeit der unteren osmotischen Resistenzgrenze vom Salzgehalt des Anpassungsmediums (AS). (Siche aud Text zu Tab. 1)

\begin{tabular}{|cccc|}
\hline \multirow{2}{*}{ RS in \%0 } & \multicolumn{3}{c|}{ Uberlebende in Prozent } \\
& AS $=5,6 \%$ & AS $=19,4 \%$ & AS $=30 \%$ \\
\hline 5,9 & & $98,0(100)$ & $97,5(113)$ \\
2,8 & & $100(179)$ & $90,0(119)$ \\
0 & $98,1(722)$ & $93,5(626)$ & $47,0(251)$ \\
\hline
\end{tabular}

Auch der zeitliche Verlauf der osmotischen Resistenzänderung in den ersten Tagen oder Stunden nach der Umsetzung in einen anderen Salzgehalt ist untersucht worden, wobei wir die Tiere aus $\mathrm{AS}=30 \%$ in $5,3 \%$ iberführten und umgekehrt. Berïcksichtigung fand dabei nur die Veränderung der oberen Resistenzgrenze. Die Geschwindigkeit der Anpassung kann bei derartigen Versuchen von der Temperatur abhängen.

Auch das Ausmaß der Salzgehaltsänderung im Außenmedium schien bei unseren Versuchen eine Bedeutung zu haben, doch wurde dies nur in einigen Vorversuchen festgestellt (vgl. hierzu Edmonds 1935 und andere). Bei den Versuchen der Tabelle 3 wählten wir zur Messung der Resistenz Konzentrationen (RS), die nach Abbildung 2 sinnvoll erschienen. Für die Tiere aus $\mathrm{AS}=30 \%$, die in $5,3 \%$ gesetzt wurden, benutzten wir RS $=48,3 \%$, denn damit kann man das Absinken der Zahl der Uberlebenden von 100 auf etwa $20 \%$ untersuchen (Abb. 3, Kurve 1). Für den umgekehrten 
Tabelle 3

Tägliche Anderung der oberen osmotischen Resistenzgrenze nach der Umsetzung. (Siehe auch T'ext zu Tab. 1)

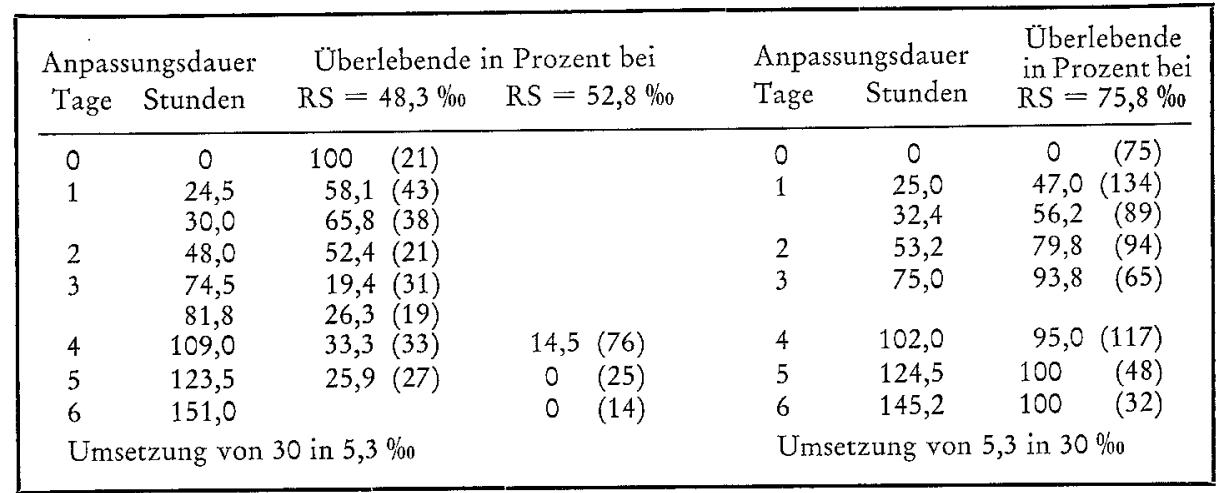

Versuch (Abb. 3, Kurve 2) konnte man das Steigen der Zahl der Uberlebenden von 0 auf gerade $100 \%$ am besten bei $R S=75,8 \%$ verfolgen. Das heißt also, wenn nach einiger Zeit noch $20 \%$ beziehungsweise gerade $100 \%$ überlebten, dann mußte die Resistenzänderung weitgehend abgeschlossen sein, was in den Versuchen nach einigen Tagen der Fall war.

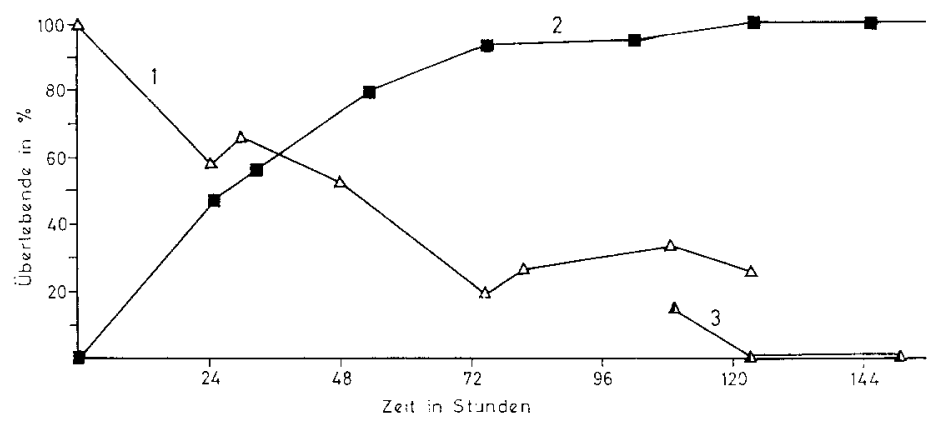

Abb. 3: Tägliche Änderung der oberen osmotischen Resistenzgrenze von Z. hiketes bei Umsetzen von 30 in 5,3\% ( 1 und 3) beziehungsweise von 5,3 in $30 \%$ (2). Salzgehalt während der Messungen (RS) bei (1): 48,3\%, bei (2): 75,8\%, bei (3): $52,8 \%$

Interessanter war die Messung der Resistenzänderung in den ersten Stunden nach der Umsetzung, die zunächst mit den gleichen Salzgehalten (RS) durchgeführt wurde (Tab. 4, 5; Abb. 4, Kurve 3 und Kurve 2). Der Abbildung 2 kann man aber entnehmen, daß diese Salzgehalte (RS) nicht richtig gewählt sind, um die Resistenzänderungen gleich von Anfang an zu erfassen. Daher wurden noch Messungen mit RS = $75,8 \%$ beziehungsweise 52,8\% durchgeführt. Aus den Kurven 4 und 1 der Abbildung 4 geht hervor, daß die Resistenzänderung nach der Umsetzung sehr schnell einsetzt. 
Wie erwähnt, werden im allgemeinen bei einer langsamen stufenweisen Anderung des Salzgehaltes im Außenmedium höhere Resistenzwerte erzielt als mit der von uns angewandten Methode. Das konnte auch für unser Versuchstier nachgewiesen werden. Die Zoothamnien wurden aus $A S=5,3 \%$ nach einem Aufenthalt von jeweils 90 Minuten in mehreren Stufen $(10,15,20$ und $25 \%$ ) schließlich in $30 \%$ überführt.
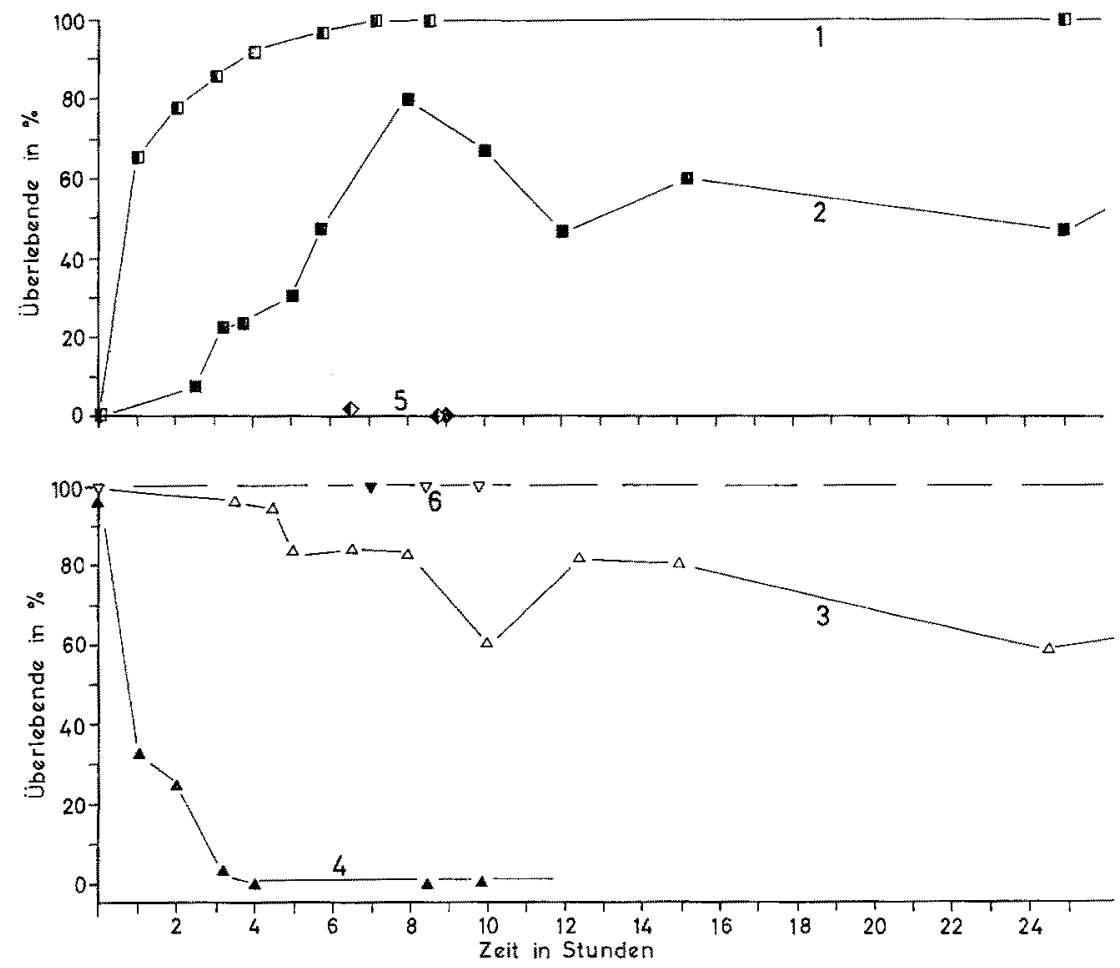

Abb. 4: Anderung der oberen osmotischen Resistenzgrenze von Z. biketes in den ersten Stunden nach Umsetzen von 5,3 in $30 \%$ (o ben) beziehungsweise von 30 in $5,3 \%$ (unten). RS bei (1) $52,8 \%$, bei (2) $75,8 \%$, bei (3) $48,3 \%$, bei (4) $75,8 \%$; (5) und (6) Kontrollwerte. Die Resistenzunterschiede, die sich nach 8 und 12 Stunden bei Unsetzung von 5,3 in $30 \%$ bei RS $=75,8 \%$ und nach 8 und 10 Stunden bei Umsetzung von 30 in $5,3 \%$ bei RS $=48,3 \%$ ergaben, können mit einer Irrtumswahrscheinlichkeit $\alpha<0,1 \%$ beziehungsweise $\alpha \approx 1 \%$ statistisch abgesichert werden (korrigierter $\chi^{2}$-Test von YATES)

Nach zwei weiteren Stunden überlebten bei RS $=52,8 \%$ von 98 Tieren $95,8 \%$; das sind $18,2 \%$ mehr als bei der direkten Uberführung. Die osmotische Resistenz war dabei also deutlich erhöht. Auch bei diesem Versuch dürfte die relativ rasch einsetzende Resistenzänderung eine Rolle gespielt haben. Die langsam überführten Tiere waren ja, bevor sie in den Salzgehalt von 30\% gebracht wurden, bereits 6 Stunden einem erhöbten Salzgehalt ausgesetzt.

Es bleibt zu diskutieren, wie der unregelmäßige Verlauf der Kurve 2 beziehungsweise 3 in Abbildung 4 zu erklären ist. Die nach dem Umsetzen anfänglich zu beobach- 
Tabelle 4

Anderung der oberen osmotischen Resistenzgrenze in den ersten Stunden nach der Umsetzung von $30 \%$ in 5,3\%. (Siehe auch Text zu Tab. 1)

\begin{tabular}{|c|c|c|c|c|}
\hline \multirow{3}{*}{$\begin{array}{l}\text { Anpassungs- } \\
\text { dauer (Std.) }\end{array}$} & \multicolumn{4}{|c|}{ Uberlebende in Prozent } \\
\hline & \multicolumn{2}{|c|}{ bei umgesetzten Tieren } & \multicolumn{2}{|c|}{ bei Kontrolltieren } \\
\hline & $\mathrm{RS}=75,8 \%$ & $\mathrm{RS}=48,3 \%$ & $\mathrm{RS}=75,8 \%$ & $\mathrm{RS}=48,3 \%$ \\
\hline 0 & $96,0(25)$ & $100 \quad(21)$ & & \\
\hline 1,0 & $32,6(86)$ & & & \\
\hline 2,0 & $25,3(74)$ & & & \\
\hline 3,2 & $2,5(80)$ & & & \\
\hline 3,5 & & $96,5(29)$ & & \\
\hline 4,0 & $0 \quad(57)$ & & & \\
\hline 4,5 & & $94,3(35)$ & & \\
\hline 5,0 & & $82,5(29)$ & & \\
\hline 6,5 & & $84,3(51)$ & & \\
\hline 7,1 & & & $100(31)$ & \\
\hline 7,4 & & & $100(38)$ & \\
\hline 8,0 & & $83,0(53)$ & & \\
\hline 8,4 & $0 \quad(11)$ & & & $100(38)$ \\
\hline 9,8 & & & & $100(26)$ \\
\hline 10,0 & & $59,4(64)$ & & \\
\hline 12,3 & & $81,5(27)$ & & \\
\hline 15,0 & & $80,9(47)$ & & \\
\hline 24,5 & & $58,1(43)$ & & \\
\hline
\end{tabular}

Tabelle 5

Anderung der oberen osmotischen Resistenzgrenze in den ersten Stunden nach der Umsetzung von 5,3 in $30 \%$. (Siehe auch Text zu Tab. 1)

\begin{tabular}{|c|c|c|c|c|c|}
\hline \multirow{3}{*}{$\begin{array}{l}\text { Anpassungs- } \\
\text { dauer (Std.) }\end{array}$} & \multicolumn{5}{|c|}{ Uberlebende in Prozent } \\
\hline & \multicolumn{2}{|c|}{ bei umgesetzten Tieren } & \multicolumn{3}{|c|}{ bei Kontrolltieren } \\
\hline & $\mathrm{RS}=52,8 \% 0$ & $\mathrm{RS}=75,8 \% 0$ & RS $=$ & $52,8 \%$ & $\mathrm{RS}=75,8 \%$ \\
\hline 0 & $1,9 \quad(53)$ & (75) & & & \\
\hline 1,0 & $65,8(149)$ & & 0 & (61) & \\
\hline 2,0 & $77,6(134)$ & & & & \\
\hline 2,5 & & $8,0 \quad(25)$ & & & \\
\hline 3,0 & $85,9 \quad(57)$ & & & & \\
\hline 3,2 & & $22,5 \quad(40)$ & & & \\
\hline 3,7 & & 23,1 (39) & & & \\
\hline 4,0 & $92,1 \quad(38)$ & & & & \\
\hline 5,0 & & $30,3 \quad(33)$ & & & \\
\hline 5,3 & $97,0 \quad(66)$ & & & & \\
\hline 5,8 & & $47,1 \quad(87)$ & & & \\
\hline 6,4 & & & 5, & $(75)$ & \\
\hline 7,2 & (50) & & & & \\
\hline 8,0 & & $80,2(101)$ & & & \\
\hline 8,6 & (34) & & & & \\
\hline 8,8 & & & 0 & $(36)$ & \\
\hline 9,1 & & & & & $O(81)$ \\
\hline 10,0 & & & & & \\
\hline 12,0 & & $46,7(169)$ & & & \\
\hline 15,3 & & $60,3(63)$ & & & \\
\hline 25,0 & (11) & $47,0(134)$ & & & \\
\hline 32,4 & & $56,2 \quad(89)$ & & & \\
\hline
\end{tabular}


tenden Volumenänderungen der Zellen wurden erwähnt. Sie werden durch einen Wassereinstrom beziehungsweise Wasserentzug bedingt, der Einfluß auf die Resistenz haben wird. Ein Wasserentzug erhöht die thermische Resistenz der Zellen (CHRIsTOPHERSEN \& PRECHT 1953), doch dürfte dies auch für die Widerstandsfähigkeit gegenüber anderen Faktoren gelten. Nach 8 bis 10 Stunden werden dann Regulationen der Zellkonzentration sichtbar, welche das Minimum beziehungsweise Maximum in den Kurven der Abbildung 4 bedingen. Es handelt sich hierbei nur um Deutungen, da wir die Zellkonzentration nicht gemessen haben. Bei größeren Änderungen des Salzgehaltes wird danach zunächst das Zellvolumen beeinflußt, da die veränderte Vakuolenleistung zur Regulation nicht ausreicht; erst nach einer gewissen Konzentrationsangleichung ist die Zelle in der Lage, die normale Größe wieder einzunehmen. Der Konzentrationsausgleich dauert an und wird im poikilosmotischen Bereich wahrscheinlich dazu führen, daß die Vakuolenfrequenz sich wie bei Frontonia auf einen Normalwert einstellt. Diese ganzen

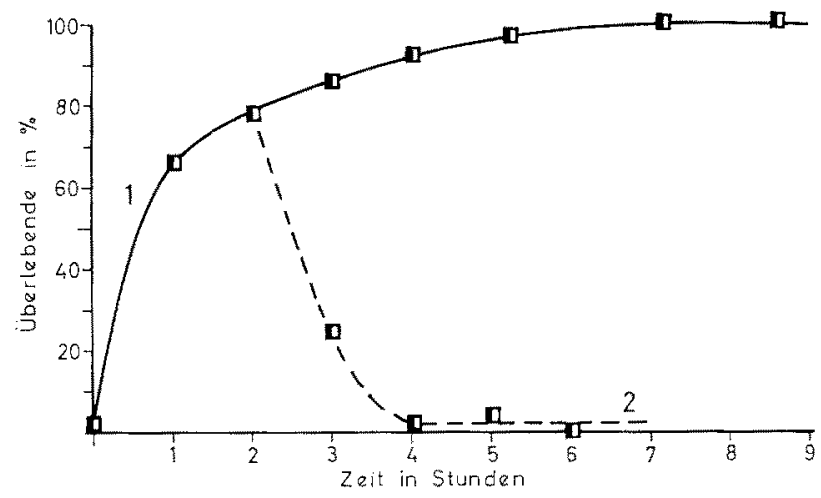

Abb. 5: Anderung der oberen osmotischen Resistenzgrenze von Z. biketes nach Umsetzung von 5,3 in $30 \%$ (RS $=52,8 \%$ ). (1) nach der Umsetzung in $30 \%$, (2) Tiere, die nach $z w e i-$ stündigem Aufenthalt in $30 \%$ nach $5,3 \%$ zurückversetzt wurden

Veränderungen, die nur mit dem langsamen Konzentrationsausgleich zusammenhängen, führen zu einer Änderung der osmotischen Resistenz. Es wird nur eine bestimmte Abweichung der Konzentration des Außenmediums von der des Innenmediums vertragen; wenn diese sich ändert, verschiebt sich auch der osmotische Toleranzbereich. Die gegebene Deutung wäre die einfachste, was nicht unbedingt besagt, daß sie richtig ist, da man eventuell schon bei den Einzellern mit hinzukommenden Adaptationen rechnen muß, wie sie auf den Seiten 364 und 366 erwähnt werden.

Vor allem Botaniker haben darauf hingewiesen, daß man eine kurzfristige Anpassung an extreme, möglichst subletale Temperaturen (ein hardening) von den langfristigen Anpassungen trennnen muß, die bisher als Resistenzadaptation bezeichnet wurden (vgl. AleXandrov 1964, Precht et al. 1966). Eine Schockanpassung, wie man das hardening bezeichnen könnte, soll auch bei Tieren vorkommen und leitet wahrscheinlich zu den stress-Phänomenen über; bei uns werden zur Zeit diesbezügliche Untersuchungen durchgeführt. Zur Prüfung der Frage, ob es auch eine Schockanpas- 
sung bei der osmotischen Resistenz gibt, überführten wir die Zoothamnien aus einem AS $=5,3 \%$ nach Bestimmung ihrer osmotischen Widerstandsfähigkeit bei RS = $52,8 \%$ in ein Medium von 30\% worden (Abb. 4, 5, Kurve 1). Die uns hier interessierenden Zoothamnien wurden nach 2 Stunden in 5,3\% zurückgesetzt, also zu einem Zeitpunkt, als bereits $77,6 \%$ der Tiere überlebten. Die Einwirkung des hohen Salzgehalts erwies sich als wenig nachhaltig, denn nach einer Stunde Aufenthalt im Ausgangsmedium war die Überlebensrate auf $24,2 \%$ (86 untersuchte Tiere) abgesunken und erreichte nach einer weiteren Stunde wieder den Ausgangswert von 1,9\% (52 Tiere, nach 5 Stunden 4,4\%, 90 Tiere; nach 6 Stunden 0\%, 22 Tiere; Abb. 5, Kurve 2).

\section{DISKUSSION}

Betrachten wir zunächst den norma le n Temperatur- und Salzgehaltbereich ohne das Resistenzproblem.

Bei einer Anderung der Versuchstemperatur gehorchen bei poikilothermen Tieren die meisten Lebensprozesse wie chemische Reaktionen der RGT-Regel (Q10-Werte = 2 bis 4). Sich anschließende Gleichgewichtseinstellungen (overshoots etc.) rechnet man sinnvollerweise ebenfalls zu den Reaktionen.

Im einfachsten Fall ist auch die osmotische Konzentrationsangleichung des Innenan das veränderte Außenmedium bei poikilosmotischen Tieren eine Reaktion. Aber schon bei unserem Versuchstier tritt dadurch eine Komplikation auf, daß Wasser schneller permeiert als dic Salze, was zu Volumenänderungen führt. Dies macht Regulationen durch bereitliegende Mechanismen, wie die kontraktile Vakuole, notwendig (Volumenregulation). Durch diese wird der alte Zustand wiederhergestellt. Die Regulation ist notwendig, weil der Konzentrationsausgleich im Gegensatz zur Temperaturangleichung bei den wechselwarmen Tieren langsam erfolgt. Auch die Befunde von OBERTHÜR (1937) an Frontonia könnten nur damit zusammenhängen und wären dann nicht als Adaptation aufzufassen.

Die poikilosmotischen Tiere wechseln zwar die Innen- mit der Außenkonzentration, doch kann manchmal die erste von der zweiten etwas abweichen. Außerdem entspricht für gewöhnlich die ionale Zusammensetzung des Innen- nicht der des Außenmediums, was durch eine Ionenregulation erreicht wird. Ist die Ionenkonzentration relativ konstant und unabhängig von der Außenkonzentration, so wird dies durch eine Osmoregulation aufrechterhalten (vgl. RemANE \& SchLIEPER 1958).

Durch eine typische Regulation, die man in Form eines Regelkreises modellmäßig darstellen kann (vgl. Hensel in: PRECHT et al. 1955, p. 342), wird die Körpertemperatur der Warmblüter konstant gehalten. Nach dem Einwirken von Störfaktoren wird durch die Regelung der Sollwert rasch wiederhergestellt. Bei einigen Tieren ist dieser Sollwert im Winterschlaf verschoben.

Auch die wechselwarmen Tiere können über Regulationen verfügen, etwa die Honigbiene, die ihre Stocktemperatur relativ konstant hält. Bei den Reptilien finden sich Anklänge zur Regulierung der Körpertemperatur.

Es bleibt zu diskutieren, wann man sinnvollerweise von Adaptationen 
(= Akklimatisationen) spricht. Nach KINNE (1964a, p. 147, 1964b) unterscheiden sich trotz mancher Übergänge Regulationen (wie die Ionen-, Volumen- und Osmoregulation bei Salzgehaltsveränderungen) im wesentlichen dadurch von Adaptationen, daßs diese echte funktionelle oder strukturelle Veränderungen im Organismus verursachen, Zeit zu ihrer "Entwicklung" benötigen und sich vielfach nicht auf spezifische Organe beschränken, sondern mehr oder minder alle Ebenen der tierischen Organisation betreffen. Regulationen dagegen haben im allgemeinen die Wiederherstellung des „Sollzustandes", aber keine echten Veränderungen des status quo ante zur Folge. Sie laufen relativ schnell $a b$ und sind das Ergebnis der routinemäßigen Aktivität spezifischer, präexistierender Reaktionssysteme. Bei den Adaptationen unterscheidet KINNE Simultanreaktionen, die Stabilisierungsphase und den neuen stationären Zustand. PRosser (1958, p. 169) trennt $z$ wischen acclimation und acclimatization: "The phase of compensation is the process of acclimation to a single environmental factor, as in controlled experiments, or of acclimatization to a complex of environmental factors as seasonal or climatic changes."

Bei den Adaptationen treten nicht nur bereitliegende Mechanismen in Tätigkeit wie die kontraktile Vakuole der Ciliaten, sondern es kommt zu grundsätzlicheren Anpassungen an einen veränderten Umweltfaktor. Neben der Struktur kann durch eine Anderung der Enzymaktivität und -quantität die Reaktionsgeschwindigkeit der Lebensprozesse verändert werden, die Zahl der Mitochondrien (JankowsKy \& KorN 1965), der Stoffwechselweg (EkBERg 1958 und andere), die Funktion von Organen oder sogar ein Regulationsmechanismus. Eine typische Akklimatisation ist die Leistungsadaptation der wechselwarmen Tiere nach Temperaturänderungen, die nach 4 Typen verlaufen kann (Typ $4=$ fehlende Adaptation; vgl. PReCHr 1961, 1964a). Bei diesen Tieren, bei denen im wesentlichen Temperaturadaptationen und keine Regulationen vorkommen, wäre der Zeitfaktor insofern zur Klassifizierung ungeeignet, als der Adaptationsmechanismus oft stark temperaturabhängig ist, so daß die Anpassung bei hohen Temperaturen relativ rasch verlaufen kann. Die Thermoregulation der Warmblüter läuft stets rasch $a b$, die hinzukommenden Adaptationen dagegen langsam. Ober diese besteht bereits eine umfangreiche Literatur (vgl. Comparative physiology of temperature regulation, 1962). Bei Hausmäusen, die BARNETT (1965) bei - $3^{\circ} \mathrm{C}$ züchtete (Kontrollen bei $21^{\circ} \mathrm{C}$ ), trat eine Stoffwechselsteigerung um das 4 fache auf. Die Nahrungsaufnahme stieg, dagegen verringerte sich die Aktivität. Auch die Größe innerer Organe und die Fortpflanzungsvorgänge bei den Weibchen waren betroffen (vgl. ferner CHAffe et al. 1963, über die Beteiligung von Hormonen Petrović et al. 1961 und andere).

Es stellt sich die Frage, was man hinsichtlich der Funktion als Osmoadaptation bezeichnen sollte. Ein Beispiel hierfür gibt Theede (1963) an, nach dem sich das Optimum der Filtrationsrate der Miesmuschel nach einer Salzgehaltsänderung zeitlich betrachtet langsam verschiebt, während der Konzentrationsausgleich relativ rasch erfolgt. Als Adaptationen muß man auch tiefgreifende strukturelle Anpassungen an den Salzgehalt bezeichnen; es ist bekannt, daß viele Tiere ihre Gestalt und ihre Lebensweise mit dem Salzgehalt ändern (Remane $\&$ Schlieper 1958, p. 28). Hingewiesen sei in diesem Zusammenhang auf die eindrucksvollen Befunde von KINNE an Cordylopbora (KINNE 1956a, b, 1958). 
Wir haben wie auch andere die Worte Adaptation und Akklimatisation als Synonyme verwandt, was für die von uns bearbeiteten Probleme durchaus möglich ist. Die Sinnesphysiologen gebrauchen das Wort Adaptation mehr für einen Regulationsmechanismus, für den das Wort Akklimatisation unpassend wäre; insofern sollte man für die hier interessierenden Phänomene dem Wort Akklimatisation den Vorrang geben, sofern Verwechslungen möglich sind.

Das deutsche Wort An passung ist unglücklicherweise mit dem Zeitfaktor verbunden. Man spricht von Anpassungen der Tiere an einen veränderten Salzgehalt, obwohl es sich um Regulationen handelt, weil sie langsam verlaufen. Dagegen würde man nicht bei der schnell verlaufenden Thermoregulation der Warmblüter von einer Anpassung sprechen, nur bei zusätzlichen Adaptationen. Das Wort Anpassung ist also wenig festgelegt.

Es bleibt die Diskussion des Resisten z problems. Bei der Resistenz gegenüber extremen Temperaturen haben wir den Terminus Adaptation sehr weit gefaßt und ihn für alle Anderungen der Kälte- und Hitzewiderstandsfähigkeit angewendet, die von der Adaptationstemperatur abhängen. Außerlich betrachtet ähneln die osmotischen Resistenzanpassungen den thermischen. Sie verlaufen wie bei $Z$. biketes meist "sinnvoll“ gegenüber beiden Grenzen. Es ist of beobachtet worden, daß die in der Nordsee vorkommenden Tiere zu höheren Salzkonzentrationen hin verschobene Grenzen zeigen als ihre Artgenossen aus der Ostsee, wobei erblich bedingte Rassenunterschiede hier nicht interessieren sollen. MCLEESE (1956) fand bei kaltadaptierten Homarus americanus (im Gegensatz zu den $15^{\circ} \mathrm{C}$ und $25^{\circ} \mathrm{C}$-Tieren) bei der unteren Salzgehaltsgrenze sogar paradoxe Phänomene (höhere Grenzkonzentration bei Tieren aus 20\% als bei solchen aus 30\% Salzgehalt).

Die Resistenzadaptation gegenüber extremen Temperaturen bei wechselwarmen Tieren ist zweifellos eine echte Akklimatisation, selbst wenn sie bei hohen Temperaturen sehr rasch verlaufen kann (vgl. PRECHT et al. 1966). Bei ihr können sogar Strukturänderungen auf der Molekülebene eine Rolle spielen. Man kann bei derartigen Untersuchungen entweder die Tiere an neue Temperaturen anpassen und die Resistenz von relativ reinen Eiweißen vor und nach der Umadaptation messen, wie etwa MEws (1957) dies bei Verdauungsfermenten von Helix pomatia tat, oder man unterzieht reine Eiweiße einer Umadaptation, wodurch sich ihre Hitzeresistenz ändern kann (Christophersen \& Thiele 1952, Christophersen 1967).

Auch bei den Warmblütern gibt es nicht nur eine Leistungs-, sondern auch eine Resistenzadaptation gegenüber extremen Temperaturen. Diese läßt sich bis auf die Zellebene verfolgen (HOLEČKOVÁ et al. 1965 und andere).

Wenn die auf Seite 362 gegebene einfache Erklärung der beobachteten Verschiebung der osmotischen Resistenzgrenzen mit dem AS bei Z. hiketes zutrifft, handelt es sich nicht um eine Adaptation, da sie dann einfach damit zusammenhängt, daß das Tier erwartungsgemäß nur eine bestimmte Abweichung der Innen- von der Außenkonzentration verträgt. $\mathrm{Da}$ die Konzentrationsangleichung bei Salzgehaltsänderungen bei poikilosmotischen Tieren im Gegensatz zur thermischen Angleichung bei wechselwarmen Tieren langsam erfolgt, erhält man das Bild einer sich über eine längere Zeit erstreckenden osmotischen Resistenzadaptation. $\mathrm{Da}$ wie erwähnt ein veränderter Wassergehalt der Zellen die Resistenz ganz allgemein beeinflussen kann (auch die 
Hitzeresistenz), ergeben sich rein äußerlich betrachtet weitere Parallelen zur Resistenzadaptation gegenüber extremen Temperaturen, bei der oft auch die allgemeine Widerstandsfähigkeit verändert wird (PRECHT 1961, 1964b). SchLIEPER und Mitarbeiter haben aber gezeigt, daß es auch echte Resistenzadaptationen an extreme Salzgehalte des Außenmediums gibt. Nach THEEDE (1965) ist bei isoliertem Kiemengewebe von Mytilus edulis, das von 30 in $15 \%$ überführt wurde, die osmotische Angleichung weit eher beendet als die Anderung der Resistenzgrenzen.

Nomenklatorische Fragen sind letztlich als zweitrangig anzusehen, doch brauchen wir zur Erfassung der so vielfältigen Naturerscheinungen eine Aufgliederung unseres Wissens; außerdem $\mathrm{zwingt}$ uns eine solche für gewöhnlich $\mathrm{zu}$ einem gründlicheren Durchdenken der Problematik.

Die Worte Adaptation und Akklimatisation beinhalten eigentlich einen Nutzen für die Art im Sinne eines Selektionsvorteils. Es wurde schon früher (Precht \& CHRISTOPHERSEN 1965) erörtert, daß es sehr viele zusätzliche Untersuchungen erfordern würde, wenn man einen solchen Nutzen in jedem Fall exakt nachweisen wollte. Im allgemeinen dürfte bei Anpassungen der intakten Tiere ein derartiger Nutzen vorliegen. Allerdings können „Begleitumstände" beobachtet werden, denen man keinen Nutzen zubilligen möchte, obwohl wir sie als Adaptation bezeichnet haben. Dies gilt beispielsweise für die mehrfach gefundene gesteigerte Hitzeresistenz frostabgehärteter Winterpflanzen (paradoxe Adaptation). Wenn ferner die Hitzeresistenz eines Zellstoffwechselprozesses in einem ganz anderen Sinne von der Adaptationstemperatur beeinflußt wird als die Widerstandsfähigkeit des intakten Tieres und wenn dies bei Versuchstemperaturen geschieht, die weit über der Letaltemperatur des 'Tieres liegen,

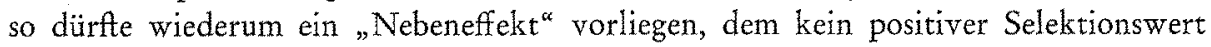
zukommt, dessen Untersuchung aber dennoch zur Aufklärung des ganzen Phänomens sehr nützlich sein kann. Im normalen Temperaturbereich gibt es etliche Beispiele für eine inverse Leistungsadaptation. Es wäre sicher falsch, dieser in jedem Fall einen Nutzen abzusprechen. Ein solcher könnte zum Beispiel der invers adaptierenden Peroxydaseaktivität der Hefe Torulopsis kefyr (CHristophersen \& Precht 1950) oder der sich ebenso verhaltenden Alkoholdehydrogenaseaktivität von Saccharomyces cerevisiae (MARQUARDT 19591) durchaus zukommen, worauf in den zitierten Arbeiten eingegangen wird. Es ist weniger wichtig, ob in den problematischen Fällen ein positiver Selektionsvorteil vorhanden ist; entscheidend ist, daß solche "Adaptationen" nicht schaden und keinen negativen Selektionswert bekommen (vgl. hierzu auch Usнakov 1964). Wir haben somit schon aus praktischen Erwägungen das komplizierte Problem des Nutzens möglichst ausgeklammert und sprechen von Adaptationen, wenn ein Wechsel der Adaptationstemperatur irgendwelche Folgeerscheinungen hat, die nicht nur als Reaktionen verstanden werden können, wie sie bei einer Anderung der Versuchstemperatur zu beobachten sind.

1 Diss. Univ. Kiel unter Anleitung von Prof. Christophersen. 


\section{ZUSAMMENFASSUNG}

1. Das Ciliat Zoothamnium biketes PRECHT ändert bei abrupten Veränderungen des Salzgehalts des Außenmediums vorübergehend das Zellvolumen.

2. Die Resistenzgrenzen gegenüber sehr niedrigen und hohen Salzgehalten hängen von der Konzentration $a b$, an welche sich die Tiere angepaßt haben.

3. Der zeitliche Verlauf der Resistenzänderung mit dem Salzgehalt des Außenmediums wird verfolgt. Nachwirkungen im Sinne eines hardening bei Temperaturänderungen konnten nicht festgestellt werden.

4. Unter Berïcksichtigung dieser Befunde wird allgemein zu den Reaktionen, Regulationen und Adaptationen ( = Akklimatisationen) der Tiere nach Veränderungen der Temperatur und des Salzgehaltes des Außenmediums Stellung genommen.

Es wurden Apparate benutzt, welche die Deutsche Forschungsgemeinschaft H. PrECHT zur Verfügung stellte.

\section{ZITIERTE LITERATUR}

Alexandrov, V. Y., 1964. Cytophysiological and cytoecological investigations of resistance of plant cells towards the action of high and low temperature. Q. Rev. Biol. 39, 35-77.

Ax, P. \& Ax, R., 1960. Experimentelle Untersuchungen über die Salzgehaltstoleranz von Ciliaten aus dem Brackwasser und Süßwasser. Biol. Zbl. 79, 6-31.

BARNETt, S. A., 1965. Adaptation of mice to cold. Biol. Reo. 40, 5-51.

Chaffe, R. R. J., Clark, R. T., Reynafarje, B., Cunningham, M. D. \& Bartlett, W. L., 1963. Some effects of cold-acclimation on the biochemistry and histology of the hamster kidney. Proc. Soc. exp. Biol. Med. 113, 115-121.

Christophersen, J., 1967. Adaptative temperature responses of microorganisms (im Druck).

- \& PRECHT, H., 1950. Fermentative Temperaturadaptation. Biol. Zbl. 69, 240-256.

- - 1953. Die Bedeutung des Wassergehaltes der Zelle für Temperaturanpassungen. Biol. Zbl. 72, 104-119.

- \& Thele, H., 1952. Uber den Einfluß von Substrat und Temperatur auf die proteolytische Aktivität einiger Käsebakterien. Kieler milchw. ForschBer, 4, 683-700.

Eomonds, E., 1935. The relations between the internal fluid of marine invertebrates and the water of the environment, with special references to Australian crustacea. Proc. Linn. Soc. N.S.W. 60, 233 247.

EKBERG, D. R., 1958. Respiration in tissues of goldfish adapted to high and low temperatures. Biol. Bull. mar. biol. Lab., Woods Hole 114, 308-316.

FENCHEL, T., 1965. On the ciliate fauna associated with the marine species of the amphipod genus Gammarus FaBRIcrus. Opbelia 2, 281-303.

HANNON, J. P. \& VIERECK, E. (Eds), 1962. Comparative physiology of temperature regulation. Arctic Aeromed. Lab., Fort Wainwright, Alaska.

HolečKová, E., Baudysová, M. \& Cinnerová, O., 1965. Adaptation of mammalian cells to cold. Resistance to cold and multiplication of $\mathcal{L}$, Detroit- 6 and HeLa cells adapted to low temperature. Expl Cell Res. 40, 396-401.

JANKOWSKY, H.-D. \& KoRN, H., 1965. Der Einfluß der Adaptationstemperatur auf den Mitochondriengehalt des Fischmuskels. Naturwissenschaften 52, 642-643.

KInNe, O., 1956a. Über Temperatur und Salzgehalt und ihre physiologisch-biologische Bedeutung. Biol. Z Zbl. 75, 314-327.

- 1956b. Über den Einfluß des Salzgehaltes und der Temperatur auf Wachstum, Form und Vermehrung bei dem Hydroidpolypen Cordylopbora caspia Pallas, Athecata, Clavidae. 1. Zool. Jb. Phys. 66, 565-638.

- 1958. Über die Reaktion erbgleichen Coelenteratengewebes auf verschiedene Salzgehaltsund Temperaturbedingungen. Zool. Jb. Pbys. 67, 407-486. 
- 1963. The effects of temperature and salinity on marine and brackish water animals. 1. Temperature. Oceanogr. mar. Biol. A. Rev. 1,301-340.

- 1964a. 2. Salinity and temperature salinity combinations. Oceanogr. mar. Biol. A. Rev. 2, 281-339.

- 1964b. Non-genetic adaptation to temperature and salinity. Helgoländer wiss. Meeresunters. 9, $433-458$.

- 1964c. Physiologische und ökologische Aspekte des Lebens in Astuarien. Helgoländer wiss. Meeresunters. 11, 131-156.

MCLEESE, D. W., 1956. Effects of temperature, salinity and oxygen on the survival of the American lobster. J. Fish. Res. Bd. Can. 13, 247-272.

Mews, H.-H., 1957. Ober die Temperaturadaptation der Sekretion von Verdauungsfermenten und deren Hitzeresistenz. Z. vergl. Physiol. 40, 345-355.

MüLLER, R., 1936. Die osmoregulatorische Bedeutung der kontraktilen Vakuolen von Amoeba proteus, Zoothamnimm biketes und Frontonia marina. Arch. Protistenk. 87, 345-382.

NELLEN, W., 1963. Fischereibiologische und faunistische Brackwasseruntersuchungen in der Schlei, einer Ostseeförde Schleswig-Holsteins. Kiel, Math.-Nat. Diss.

OBERTHÜr, K., 1937. Untersudhungen an Frontonia marina FABRE-DoM. aus einer BinnenlandSalzquelle unter besonderer Berüdssichtigung der pulsierenden Vakuole. Arch. Protistenk. 88, 387-420.

OHL, H., 1959. Temperatur- und Salzgehaltsmessungen an der Oberfläche des Kieler Hafens in den Jahren 1952-1957. Kieler Meeresforsch. 15, 157.

Petrović, V., Andjus, R. K. \& Dukrć, S., 1961. Rôle de la thyroide et des surrénales dans l'adaptation thermique du rat. Arb. biol. Nanka 13, 7-20.

Precht, H., 1935. Epizoen der Kieler Bucht. Nova Acta Leopoldina, N. F., 3, 405-474.

- 1961. Temperaturanpassungen bei wechselwarmen Tieren. Zool. Anz. (Suppl. Bd) 24, 38-60.

- 1963. Anpassungen wechselwarmer Tiere zum Uberleben extremer Temperaturen. Naturwiss. Rdsch. 16, 9-16.

- 1964a. Anpassungen wechselwarmer Tiere im normalen Temperaturbereich und ihre Ursachen. Naturwiss. Rdsch. 17, 438-442.

- 1964b. Uber die Resistenzadaptation wechselwarmer Tiere an extreme Temperaturen und ihre Ursachen. Helgoländer wiss. Meeresunters. 9, 392-411.

- Basedow, T., Bereck, R., Lange, F., Thiede, W. \& Wilke, L., 1966. Reaktionen und Adaptationen poikilothermer Tiere nach einer Anderung der Anpassungstemperatur und der zeitliche Verlauf. Helgoländer wiss. Meeresunters. 13,369-401.

- \& Chrtstophersen, J., 1965. Temperaturadaptation des Cilienepithels isolierter Kiemen und Fühlerspitzen von Mollusken. Z. wiss. Zool. 17, 197-209.

- - \& Hensel, H., 1955. Temperatur und Leben. Springer, Berlin, $514 \mathrm{pp}$.

Prosser, C. L. (Ed.), 1958. Physiological adaptation. A symposium held ... at the Mar. Biol. Lab., Woods Hole, Mass., Sept. 5-7, 1957. Am. Physiol. Soc., Washington, DC., 158 pp.

- \& Brown, F. A., 1961. Comparative animal physiology. 2nd ed. Saunders, Philadelphia, $\mathrm{Pa}, 688 \mathrm{pp}$.

Remane, A. \& Schlieper, C., 1958. Die Biologie des Brackwassers. Schweizerbart, Stuttgart, $348 \mathrm{pp}$. (Binnengewässer 22.)

SCHLIEPER, C., 1960. Genotypische und phänotypische Temperatur- und Salzgehaltsadaptationen bei marinen Bodenevertebraten der Nord- und Ostsee. Kieler Meeresforsch. 16, 180-185.

THEEDE, H., 1963. Experimentelle Untersuchungen über die Filtrierleistung der Miesmuschel Mytilus edulis L. Kieler Meeresforsch. 19, 20-41.

- 1965. Vergleichende experimentelle Untersuchungen über die zelluläre Gefrierresistenz mariner Muscheln. Kieler Meeresforsch. 21, 153-166.

Ushakov, B., 1964. Thermostability of cells and proteins of poikilotherms and its significance in speciation. Physiol. Rev. 44, 518-560.

Vogel, W., 1966. Ưber die Hitze- und Kälteresistenz von Zoothamnium biketes PrechT (Ciliata, Peritricha). Z. wiss. Zool. 173, 344-378.

WERER, E., 1961. Grundriß der biologischen Statistik für Naturwissenschafler, Landwirte und Mediziner. 4. Aufl. G. Fischer, Jena, 566 pp. 